\title{
Risk factors associated with stillbirth of piglets born from oxytocin-assisted parturitions
}

\author{
Nguyen Hoai Nam¹ ${ }^{1}$ and Peerapol Sukon 2,3 (D) \\ 1. Department of Animal Surgery and Theriogenology, Faculty of Veterinary Medicine, Vietnam National University of \\ Agriculture, Trauqui, Gialam, Hanoi, Vietnam; 2. Department of Anatomy, Faculty of Veterinary Medicine, Khon Kaen \\ University, Mueang Khon Kaen District, Khon Kaen 40002, Thailand; 3. Research Group for Animal Health Technology, \\ Khon Kaen University, Mueang Khon Kaen District, Khon Kaen 40002, Thailand. \\ Corresponding author: Nguyen Hoai Nam, e-mail: hoainam26061982@yahoo.com \\ Co-author: PS: sukonp@kku.ac.th \\ Received: 29-05-2020, Accepted: 04-09-2020, Published online: 17-10-2020
}

doi: www.doi.org/10.14202/vetworld.2020.2172-2177 How to cite this article: Nam NH, Sukon P (2020) Risk factors associated with stillbirth of piglets born from oxytocin-assisted parturitions, Veterinary World, 13(10): 2172-2177.

\begin{abstract}
Aim: The present study aimed to investigate the effects of different risk factors on stillbirth of piglets born from oxytocinassisted parturitions.

Materials and Methods: Data were collected from a total of 1121 piglets born from 74 Landrace x Yorkshire crossbred sows from a herd. Logistic regression models were used to determine the associations between stillbirth and different risk factors including parity (1, 2, 3-5, and 6-10), gestation length (GL) (112-113, 114-116, and 117-119 days), litter size, birth order (BO), sex, birth interval (BI), cumulative farrowing duration, birth weight (BW), crown rump length, BW deviation, body mass index, ponderal index (PI), and the use of oxytocin during expulsive stage of farrowing.
\end{abstract}

Results: The incidence of stillbirth at litter level and stillbirth rate was 59.5\% (44/74) and 8.1\% (89/1094), respectively. The final multivariate logistic regression selected BO, BI, PI, GL, and parity as the five most significant risk factors for stillbirth. Increased $\mathrm{BO}$ and $\mathrm{BI}, \mathrm{GL}<114$ and $>116$ days, parity 6-10, and low PI increased the stillbirth rate in piglets.

Conclusion: Several factors previously determined as risks for stillbirth in exogenous oxytocin-free parturitions also existed in exogenous oxytocin-assisted parturitions. One dose of oxytocin at fairly high BO did not increase stillbirth, whereas two doses of oxytocin were potentially associated with increased values.

Keywords: birth order, oxytocin, pig, ponderal index, stillbirth.

\section{Introduction}

Oxytocin is commonly used as an obstetric intervention to reduce farrowing duration in sows [1], with controversial effects on stillbirth rates. Some authors did not find any correlation between the use of oxytocin and stillbirth incidents [2-5], while other researchers reported that oxytocin reduced stillbirth rates [6]. On the other hand, there are many studies that found increased stillbirths due to oxytocin-induced asphyxia [7-11].

Studies that evaluated the effect of oxytocin on stillbirth at litter level $[4,5,12]$ might include some degree of bias since stillborn piglet(s) might be born before the use of oxytocin. At the same time, evaluation of effect of oxytocin on stillbirth is difficult when it is combined with manual extraction $[4,12,13]$. Administration of oxytocin after the birth of the first piglet resulted in either increased hypoxia and stillbirth [7-11] or non-significant effect [14]. Although those studies significantly contributed in the understanding of oxytocin action in uterus and of its effect

Copyright: Nam and Sukon. Open Access. This article is distributed under the terms of the Creative Commons Attribution 4.0 International License (http://creativecommons.org/licenses/ by/4.0/), which permits unrestricted use, distribution, and reproduction in any medium, provided you give appropriate credit to the original author(s) and the source, provide a link to the Creative Commons license, and indicate if changes were made. The Creative Commons Public Domain Dedication waiver (http:// creativecommons.org/publicdomain/zero/1.0/) applies to the data made available in this article, unless otherwise stated. on stillbirth, the administration of oxytocin at the very early stages of parturition did not resemble the real practice at farrowing condition. There is also a study that examines the relationship between oxytocin use at different birth order (BO) (after 1, 4, and 8) and stillbirth incidents [15], but the interaction of oxytocin use with other factors was not evaluated despite the fact that stillbirth has a multifactorial cause. To the best of our knowledge, a study assessing the effect of sow, farrowing, and piglet traits on stillbirth of piglets born from exogenous oxytocin-assisted farrowed sows has not been reported.

The aim of this study was to investigate the effects of different factors including parity, gestation length (GL), litter size (LS), piglet gender, birth interval (BI), cumulative farrowing duration (CFD), BO, crown rump length (CRL), birth weight (BW), birth weight deviation (BWD), body mass index (BMI), and ponderal index (PI) on the stillbirth of piglets born from exogenous oxytocin-assisted parturitions.

\section{Materials and Methods}

\section{Ethical approval}

This study did not use animal samples or involve activities that could harm the investigated animals.

\section{Study period, location, animals and housing}

This study was conducted from February to May 2019; included a total of 1121 piglets born from 
74 sows on a swine farm in Phu Tho province in the North of Vietnam. The farm had a breeding herd of 600 sows. During gestation, sows were housed in individual crates sized $60 \mathrm{~cm}$ width $\times 220 \mathrm{~cm}$ length. Approximately a week before the estimated farrowing date, animals were removed to individual farrowing crates sized $180 \mathrm{~cm}$ width $\times 220 \mathrm{~cm}$ length. At farrowing crates animals were allocated into a slatted floor area which measured $60 \mathrm{~cm} \times 220 \mathrm{~cm}$. The farrowing crates were cleaned twice per day. Sows were bathed once per day or every 2 days depending on the ambient temperature. The temperature at the farrowing room ranged between $20^{\circ} \mathrm{C}$ and $26^{\circ} \mathrm{C}$.

Sows were fed 3 times/day. During the first 84 days of gestation, sows were provided with 1.8-2.5 kg of feed per day containing $13 \%$ of crude protein and metabolizable energy of $2900 \mathrm{kcal} / \mathrm{kg}$ (Hi-Gro 566F, Charoen Pokphand, Vietnam). In the last trimester, sows were daily received $3-3.5 \mathrm{~kg}$ of feed containing $17 \%$ crude protein and metabolizable energy of $3100 \mathrm{kcal} / \mathrm{kg}$ (Hi-Gro 567SF, Charoen Pokphan, Vietnam). At parturition, sows were deprived of feed. After parturition, amount of feed was increased gradually from $1.5 \mathrm{~kg}$ on day 1 to about $6.5 \mathrm{~kg}$ on day 6 . Water was provided $\mathrm{ad}$ libitum through a bite nipple system.

\section{Data collection}

Sows were supervised continuously at least from the birth of the first piglet to the birth of the last piglet. All the born piglets were handled by one veterinarian. Parity number was recorded from sow's card. GL was the interval between the date of the first insemination and the farrowing date. At the birth of each piglet the sex of piglet, birth time and $\mathrm{BO}$ were recorded. The BI (min) was defined as the interval between the births of two successive piglets, and therefore the first piglets did not have a BI. The CFD ( $\mathrm{min}$ ) was calculated as the interval between the birth of a given piglet and the birth of the first piglet, the CFD of the first piglets of all litters was 0 . Stillbirth examination was conducted immediately after the piglet expulsion. Stillborn piglets were defined as piglets died before the expulsion without any sign of decay [3]. Stillbirth rate was the proportion of all stillborn piglets to the sum of born alive and stillborn from all litters. Birth LS was calculated as the total number of born alive, stillborn, and mummified piglets. Mucus was suckled from piglets' mouth and nose, and their bodies were dried by hygroscopic flour (Safeguard, Greenvet, Vietnam). Piglets' BW $(\mathrm{kg})$ was measured individually using a $5 \mathrm{~kg}$ scale (Nhon Hoa, Vietnam). CRL $(\mathrm{cm})$ of piglets was measured using a tape measure (Vietnam). The measurement of BW and CRL was handled gently to minimize stress, and lasted about 35-40s for each piglet. BWD $(\mathrm{kg})$ was defined as the difference between $\mathrm{BW}$ of a given piglet and the mean of individual BW of piglets (alive and stillborn) in that litter. Piglets' BMI was calculated using the following equation: BW $(\mathrm{kg}) /(\mathrm{CRL}, \mathrm{m})^{2}[16]$. Piglets' PI was calculated using the following equation: $\mathrm{BW}(\mathrm{kg}) /(\mathrm{CRL}, \mathrm{m})^{3}$ [16]. During the expulsive stage, all the sows were injected 1-2 doses of oxytocin (20UI, CP-CIN 20, L.B.S. Laboratory L.T.D., Thailand) by farm's workers. The veterinarian recorded the BO at which oxytocin was used.

\section{Statistical analysis}

Descriptive statistics were generated using all available data of sows and piglets. Risk analysis was conducted in piglets with full records resulting in removal of the 74 first-born piglets that did not have a BI, 27 mummified piglets and 25 piglets with missed information in other criteria. Consequently, 995 piglets with the full record were included in risk analyses. Potential risk factors for stillbirth included oxytocin use (piglets born before oxytocin administration [O_0], born after one dose of oxytocin [O_1], and born after 2 doses of oxytocin [O_2]), parity $(1,2,3-5$, and 6-10), GL (112-113, 114-116, and 117-119 days), $\mathrm{LS}, \mathrm{BO}$, piglet gender, CRL, BW, BWD, BI, CFD, BMI, and PI. The binary dependent variable was stillbirth status of individual piglet which was categorized into No stillbirth or Stillbirth.

The relationship between 13 potential risk factors for stillbirth was analyzed using Pearson's bivariate correlation (IBM SPSS Statistics for Windows, Version 22.0, IBM Corp., Armonk, NY, USA).

A two-step modeling approach was used to determine potential risk factors associated with stillbirth. At first, a univariate logistic analysis was performed to quantify the correlation between stillbirth status and each risk factor. Second, the variables with $\mathrm{p} \leq 0.1$ in the univariate analysis were further analyzed in the multivariate analysis. A forward stepwise model building method using Akaike information criterion as the calibration tool was employed to select the final model. All statistical analyses were carried out in STATA, version 15 (StataCorp LP, College Station, TX: StataCorp LLC). A $p \leq 0.05$ was set as the statistical significance level in the final model.

\section{Results}

Forty-four (59.5\%) out of 74 litters had at least one stillborn piglet. Among them, 22 litters had 1 stillbirth, 12 litters had 2 stillbirths, and 10 litters had more than 2 stillbirths. Among the 1121 piglets born to 74 litters, 27 piglets were mummified $(2.4 \%)$ and 89 piglets were stillborn $(8.1 \%, 89 / 1094)$. Sixty-four $(86.5 \%)$ sows were injected with 1 dose of oxytocin, and $10(13.5 \%)$ sows were injected with two doses of oxytocin during the stage of fetal expulsion. The average and median $\mathrm{BO}$ at which the first and the second dose of oxytocin was injected was $8.2 \pm 3.7$ and 8 , and $10.9 \pm 2.9$ and 11 , respectively. Other parameters of investigated sows and piglets are shown in Table- 1 .

The significant correlations between significant risk factors for stillbirth are demonstrated in Table-2.

Univariate analysis results of potential risk factors for stillbirth in piglets are presented in Table-3. All 
risk factors, apart from sex and CRL, were significantly associated with stillbirth.

Table-1: Descriptive parameters of investigated sows and piglets.

\begin{tabular}{lcc}
\hline Parameters & Number & Mean \pm SD \\
\hline Parity & 74 & $4.6 \pm 2.2$ \\
GL & 74 & $115.4 \pm 1.2$ \\
LS & 74 & $15.8 \pm 3.1$ \\
CRL & 1081 & $27.0 \pm 2.6$ \\
BW & 1072 & $1.27 \pm 0.44$ \\
BI & 1013 & $19.0 \pm 41.7$ \\
CFD & 1087 & $149.5 \pm 141.9$ \\
BMI & 1072 & $17.1 \pm 4.7$ \\
PI & 1072 & $63.3 \pm 17.2$ \\
BWD & 1072 & $0.0003 \pm 0.36886$ \\
\hline
\end{tabular}

$\mathrm{SD}=$ Standard deviation, $\mathrm{GL}=$ Gestation length (day), $\mathrm{LS}=$ Litter size, $\mathrm{CRL}=$ Crown rump length $(\mathrm{cm}), \mathrm{BW}=$ Birth weight $(\mathrm{kg}), \mathrm{BI}=$ Birth interval $(\mathrm{min}), \mathrm{CFD}=$ Cumulative farrowing duration $(\mathrm{min}), \mathrm{BMI}=$ Body mass index, $\mathrm{PI}=$ Ponderal index, $\mathrm{BWD}=$ Birth weight deviation $(\mathrm{kg})$
The final model, with an overall significant value of 0.001 , showed that BO, PI, BI, GL, and parity were the five most important risk factors with respect to stillbirth in the piglets (Table-4). The final model explained $21.53 \%$ variation of stillbirth in piglets. BO was the most significant factor explaining $8.34 \%$ variation of stillbirth. PI, BI, GL, and LS explained $3.42,3.00,1.84$, and $4.93 \%$ variation of stillbirth, respectively. The Hosmer-Lemeshow goodness of fit test showed good fit between expected and predicted outcome $(\mathrm{p}=0.778)$.

The final model rejected CFD and $\mathrm{O}$ in favor of $\mathrm{BO}$ to be a better indicator regarding stillbirth. In all models containing both $\mathrm{BO}$ and $\mathrm{O}$, the stillbirth rate in piglets born before the use of oxytocin was not different from that in piglets born after the use of 1 dose of oxytocin, but different from that in piglets born after the use of 2 doses of oxytocin. Piglets with lower BW and BMI were more likely to be stillborn than those

Table-2: Matrix of significant correlations between the significant risk factors regarding stillbirth.

\begin{tabular}{|c|c|c|c|c|c|c|c|c|c|}
\hline & LS & BW & BWD & PI & BMI & BO & BI & CFD & 0 \\
\hline BW & $-0.205^{* *}$ & & & & & & & & \\
\hline BWD & & $0.834 * *$ & & & & & & & \\
\hline PI & $-0.112 * *$ & $0.670 * *$ & $0.537 * *$ & & & & & & \\
\hline BMI & $-0.162 * *$ & $0.875 * *$ & $0.718 * *$ & $0.944 * *$ & & & & & \\
\hline BO & $0.328 * *$ & & 0.080* & $-0.071 *$ & & & & & \\
\hline BI & $-0.064 *$ & & & & & $-0.109 * *$ & & & \\
\hline CFD & $0.172 * *$ & $-0.064 *$ & & $-0.174 * *$ & $-0.141 * *$ & $0.469 * *$ & $0.256 * *$ & & \\
\hline $\mathrm{O}$ & $0.195 * *$ & & & $-0.127 * *$ & $-0.092 * *$ & $0.650 * *$ & & $0.582 * *$ & \\
\hline $\mathrm{P}$ & $0.075^{*}$ & & & & & & & $-0.064 *$ & $-0.075^{*}$ \\
\hline GL & & & & & & & & $-0.150 * *$ & \\
\hline
\end{tabular}

$\mathrm{LS}=$ Litter size, $\mathrm{BW}=$ Birth weight, $\mathrm{BWD}=$ Birth weight deviation, $\mathrm{PI}=$ Ponderal index, $\mathrm{BMI}=\mathrm{Body}$ mass index, $\mathrm{BO}=\mathrm{Birth}$ order, $\mathrm{BI}=$ Birth interval, $\mathrm{CFD}=$ Cumulative farrowing duration, $\mathrm{O}=$ Use of oxytocin, $\mathrm{P}=$ Parity, $\mathrm{GL}=$ Gestation length.

$*$ means the correlation is significant at the 0.05 level. ** means the correlation is significant at the 0.01 level

Table-3: Results of univariate analysis of risk factors for stillbirth (995 piglets).

\begin{tabular}{|c|c|c|c|c|}
\hline Covariates & Stillbirth rate & OR; 95 CI & Nagelkerke R2 & p-value \\
\hline Born before the use of $\mathrm{O}$ & $3.9(20 / 511)$ & 1 & 0.0791 & \\
\hline Born after 1 dose of 0 & $10.5(45 / 433)$ & $2.85 ; 1.65-4.90$ & & $<0.001$ \\
\hline Born after 2 doses of $\mathrm{O}$ & $31.4(16 / 51)$ & $11.22 ; 5.35-23.56$ & & $<0.001$ \\
\hline Parity $6-10$ & $11.7(42 / 360)$ & 1 & 0.0285 & \\
\hline Parity 2 & $1.2(1 / 86)$ & $0.09 ; 0.01-0.66$ & & 0.018 \\
\hline Parity 1 & $5.0(6 / 121)$ & $0.40 ; 0.16-0.95$ & & 0.039 \\
\hline Parity 3-5 & $7.5(32 / 428)$ & $0.61 ; 0.10-0.18$ & & 0.046 \\
\hline $\mathrm{GL}=114-116$ days & $6.9(52 / 751)$ & 1 & 0.0155 & \\
\hline $\mathrm{GL}=117-119$ days & $9.4(16 / 170)$ & $1.40 ; 0.78-2.51$ & & 0.265 \\
\hline $\mathrm{GL}=112-113$ days & $17.6(13 / 74)$ & $2.86 ; 1.48-5.55$ & & 0.002 \\
\hline Female & $8.0(36 / 450)$ & 1 & $<0.001$ & \\
\hline Male & $8.3(45 / 545)$ & $1.035 ; 0.66-1.63$ & & 0.883 \\
\hline LS & & $1.08 ; 1.007-1.16$ & 0.082 & 0.032 \\
\hline BO & & $1.19 ; 1.13-1.25$ & 0.0834 & $<0.001$ \\
\hline BI & & $1.007 ; 1.003-1.01$ & 0.0239 & $<0.001$ \\
\hline CFD & & $1.004 ; 1.003-1.006$ & 0.0594 & $<0.001$ \\
\hline BW & & $0.92 ; 0.88-0.97$ & 0.0167 & 0.002 \\
\hline CRL & & $1.02 ; 0.93-1.11$ & 0.0003 & 0.681 \\
\hline BWD & & $0.39 ; 0.21-0.70$ & 0.0174 & 0.002 \\
\hline BMI & & $0.90 ; 0.85-0.94$ & 0.0352 & $<0.001$ \\
\hline PI & & $0.97 ; 0.95-0.98$ & 0.042 & $<0.001$ \\
\hline
\end{tabular}

$\mathrm{OR}=$ Odds ratio, $\mathrm{CI}=$ Confident interval, $\mathrm{p}=$ Probability level, $\mathrm{O}=$ Oxytocin, $\mathrm{GL}=$ Gestation length, $\mathrm{LS}=\mathrm{Litter}$ size, $\mathrm{BO}=\mathrm{Birth}$ order, $\mathrm{BI}=$ Birth interval, $\mathrm{CFD}=$ Cumulative farrowing duration, $\mathrm{BW}=$ Birth weight, $\mathrm{CRL}=$ Crown rump length, $\mathrm{BWD}=\mathrm{Birth}$ weight deviation, $\mathrm{BMI}=$ Body mass index, $\mathrm{PI}=$ Ponderal index 
Table-4: Results of multivariate analysis of risk factors for stillbirth (995 piglets).

\begin{tabular}{lccc}
\hline Stillbirth & OR; 95\%CI & Nagelkerke R2 change & p-value \\
\hline BO & $1.21 ; 1.14-1.28$ & 0.0834 & $<0.001$ \\
PI & $0.96 ; 0.94-0.98$ & 0.1176 & $<0.001$ \\
BI & $1.01 ; 1.00-1.01$ & 0.1476 & $<0.001$ \\
GL=114-116 days & 1 & 0.1660 & 0.024 \\
GL=117-119 days & $2.12 ; 1.10-4.08$ & & $<0.001$ \\
GL=112-113 days & $5.50 ; 2.50-12.07$ & 0.2153 & 0.001 \\
Parity=6-10 & 1 & & 0.021 \\
Parity=2 & $0.03 ; 0.004-0.26$ & \\
Parity=1 & $0.32 ; 0.12-0.84$ & & 0.004 \\
Parity=3-5 & $0.46 ; 0.27-0.78$ & & \\
\hline
\end{tabular}

$\mathrm{OR}=$ Odds ratio, $\mathrm{CI}=$ Confident interval, $\mathrm{p}=$ Probability level, $\mathrm{BO}=$ Birth order, $\mathrm{PI}=$ Ponderal index, $\mathrm{BI}=$ Birth interval, $\mathrm{GL}=$ Gestation length. The final model selected BO, PI, BI, GL, and parity as the five most significant factors for stillbirth in piglets. The overall significance of final model was 0.001 . The final model explained $21.53 \%$ variation of the stillbirth. The Hosmer-Lemeshow goodness of fit test showed a good fit between expected and predicted outcome $(p=0.778)$

with higher BW and BMI, respectively. However, PI was more significant than BW and BMI with respect to explanation of stillbirth variation and was retained in the final model. PI was negatively associated with stillbirth $(p<0.001)$, whereas increased BI resulted in increased stillbirth $(\mathrm{p}=0.001)$. Piglets born from sows with a GL of 114-116 days were less likely to be stillborn in comparison with piglets born from sows with a GL of 112-113 days $(\mathrm{p}<0.001)$ and $117-119$ days $(\mathrm{p}=0.024)$. Piglets born from sows at parity 1,2 , and 3-5 had lower possibility of being stillborn compared to piglets born from sows at parity $6-10(\mathrm{OR}=0.32$ $[\mathrm{p}=0.021], \quad \mathrm{OR}=0.03 \quad[\mathrm{p}=0.001], \quad$ and $\quad \mathrm{OR}=0.46$ $[\mathrm{p}=0.004])$, respectively.

\section{Discussion}

The stillbirth rate $(8.1 \%)$ found in this study is higher than that previously reported by many other authors [14,17-20]. The proportion of litters having stillbirth(s) $(59.5 \%)$ was also higher than that in any previously published articles [3,5,17,21].

The direct relationship between stillbirth and $\mathrm{BO}$ found in the present study is in accordance with the existing literature [16,22]. This study also confirms the finding of Baxter et al. [23] that BO is more important than CFD in prediction of stillbirth. It was reported that the stillbirth rate was increased from $2 \%$ in the first-born piglets to $17 \%$ in the piglets born $13^{\text {th }}$ or later [24]. The result was quite similar to the findings of the present study where stillbirth rate was $3.4 \%$ till the birth of the $5^{\text {th }}$ piglet, and increased up to $12.8 \%$ between the $11^{\text {th }}$ and $15^{\text {th }}$ piglet and to $21.4 \%$ after the $15^{\text {th }}$ piglet. Piglets with a long CFD did not necessarily have a high $\mathrm{BO}$, therefore, did not necessarily experience a high number of uterine contraction series. In contrast, piglets with a high $\mathrm{BO}$ were more likely to be subjected to an increased number of uterine contraction series, thereby being predisposed to higher risks of distress and hypoxia and subsequently being stillbirth.

The present study also confirms previously published results $[16,22]$ that $\mathrm{BI}$ is an important indicator of stillbirth in piglets. The finding that BI is more important than CFD regarding stillbirth is in agreement with that of Baxter et al. [22]. On the other hand, Langendijk et al. [25] suggested that interventions aiming at reducing stillbirth rate should focus on CFD rather than BI because the stillbirth risk significantly increased only when a BI exceeded $90 \mathrm{~min}$, whereas the stillbirth possibility started to increase when a CFD exceeded 120 min. However, in the present study, the risk of stillbirth significantly elevated at a $\mathrm{BI}>60 \mathrm{~min}(7.5 \%, 7.1 \%, 8.3 \%$, and $20.4 \%$ at $\mathrm{BI}$ $<20,21-40,41-60$, and $>60 \mathrm{~min}$, respectively), and at a CFD $>300 \min (4.7 \%, 8.1 \%, 7.7 \%$, and $21.5 \%$ at CFD $<120,121-240,241-300$, and $>300 \mathrm{~min}$, respectively). At present, there are no clear explanations for the increased importance of BI over CFD regarding stillbirth prediction. BI more suitably represents the time period that a given piglet actively manages to go through the cervix and vagina to the outside environment, whereas CFD represents the cumulative time period a given piglet is less active in the uterus plus a BI. There is no information about the different pressure levels that a piglet has to experience at different locations of the birth canal. However, based on the superior tension and thickness of the cervix and its smaller size in comparison with uterus and vagina, and its inherent long size, it can be hypothesized that a fetus experienced the highest pressure when it is pushed through the cervix. Alonso-Spilsbury et al. [26] reported that injection of oxytocin after the birth of the first piglet resulted in $70.8 \%, 8.3 \%$, and $20.8 \%$ stillbirths at BO $<5,5-8$, and $\geq 9$, respectively. This result implied the increased important role of BI compared with that of CFD in explaining stillbirth, and that may be a possible explanation to the selection of BI rather than CFD in the final model with respect to stillbirth prediction.

Body size and shape have been widely recognized as some of the most important indicators for piglet stillbirth $[16,22,23]$. The previous studies suggested that PI and BMI were more important indices than BW and CRL $[16,23]$. The present study also confirmed that PI, among conformation traits, was the most significant factor with respect to stillbirth. Piglets with a low PI tend to have a low BW with 
either a normal or a longer-than-normal CRL. The disproportionate piglets were suggested to be suffered from uterine growth retardation [27]; therefore, they did not have good health to achieve a good chance of prenatal survival. Furthermore, the proportionately small piglets have lower hemoglobin level in comparison with heavier littermates [28]. During farrowing process, distress, and hypoxia are not uncommon [8]; therefore, the lower hemoglobin level becomes a disadvantage for the small piglets and as the consequence, they were more likely to be stillborn.

There are controversies over the effect of GL on stillbirth of piglets. Some authors did not detect any effect of GL on stillbirth [29]. In contrast, the negative association between short GL and stillbirth has been reported [17]. Piglets born from a short GL associated with immaturity [28] and, therefore, acquired higher risk of being stillbirth. On the other hand, increased stillbirth in human has been also found in prolonged gestation [30-32], and this has been suggested to be attributable to placental insufficiency [33]. Therefore, the increased possibility of delivering stillborn piglets by sows with $\mathrm{GL}=117-119$ days may be an effect of placental insufficiency attributable to prolonged gestation.

The observed effect of parity number on stillbirth, in this study, was highly similar to that of Vanderhaeghe et al. [34]. In that study, the stillbirth rate in piglets born from sows at parity 1, 2, 3-6, and $>6$ was $6.8,6.1,7.4$, and $10.3 \%$, respectively. It was suggested that prolonged farrowing was the reason of increased stillbirth rates in high parity [5]. This fact was also demonstrated in the present study because BI and CFD of piglets born from sows at their $6^{\text {th }}-10^{\text {th }}$ parity were significantly longer than that of piglets born from 1,2 , and $3-5$ parity sows (22.1 versus $16.4,15.6$, and $17.7 \mathrm{~min}[\mathrm{BI}]$, and 176.6 versus $113.5,126.6$, and 158.0 min $[\mathrm{CFD}])$.

The fact that the effect of the single dose of oxytocin on stillbirth rate found in univariate analysis disappeared in all the multivariate models containing $\mathrm{BO}$ and $\mathrm{O}$ suggested that the association between the use of one dose of oxytocin and stillbirth was the result of increased BO instead of oxytocin use itself. The harmful effect of exogenous oxytocin on stillbirth has been reported in previous studies [7,9-12], where oxytocin was administered immediately after the birth of the first piglets. At early parturition, the use of oxytocin may produce uterine hyperstimulation resulting in severe fetal distress and asphyxia [15]. In contrast, oxytocin administration after the birth of the $8^{\text {th }}$ piglet resulted in the improvement of the piglet survival through sufficient stimulation of uterine contraction without considerably decreased uterine blood flow [15]. In this study, oxytocin-hyperstimulation might not be induced since oxytocin was used at fairly high BOs $(8.2 \pm 3.7$ with median $=8$ for the first dose $)$, which may be a possible explanation to the non-significant difference in stillbirth rate between piglets born before and after the use of 1 dose of oxytocin. On the other hand, the rejection of $\mathrm{O}$ in favor of other factors in the final model did not exclude potential effect of the use of two doses of oxytocin on stillbirth since this effect was significant in all models even with the presence of both $\mathrm{BO}$ and $\mathrm{O}$. This effect can be explained by the fact that piglets born late in the farrowing process were subjected to a longer period of distress and hypoxia, and at very late parturition even mild distress and hypoxia caused by the second oxytocin injection could lead to an increased risk of fetal death.

\section{Conclusion}

This study confirmed that several established risk factors for stillbirth in exogenous oxytocin-free parturitions also existed in exogenous oxytocin-assisted-parturitions. Increased BO and BI, parity 6-10, GL of 112-113 and 117-119 days, and low PI were the most significant factors associated with increased stillbirth. Injection of one dose of oxytocin at fairly high BO did not cause any significant effect on stillbirth; however, using two doses of oxytocin during the expulsive stage potentially increased stillbirth.

\section{Authors' Contributions}

NHN collected the data. NHN and PS conceived and designed the study, analyzed data, interpreted results, and wrote the manuscript. Both authors read and approved the final manuscript.

\section{Acknowledgments}

The authors are grateful to Dang Thi Hong Nhung for her assistance in data collection. This research did not receive any specific grant from funding agencies in the public, commercial, or not-for-profit sectors.

\section{Competing Interests}

The authors declare that they have no competing interests.

\section{Publisher's Note}

Veterinary World remains neutral with regard to jurisdictional claims in published institutional affiliation.

\section{References}

1. Kirkden, R.D., Broom, D.M. and Andersen, I.L. (2013) Invited review: Piglet mortality: Management solutions. $J$. Anim. Sci., 91(7): 3361-3389.

2. Vanderhaeghe, C., Dewulf, J., Ribbens, S., de Kruif, A. and Maes, D. (2010b) A cross-sectional study to collect risk factors associated with stillbirths in pig herds. Anim. Reprod. Sci., 118(1): 62-68.

3. Vanderhaeghe, C., Dewulf, J., De Vliegher, S., Papadopoulos, G.A., de Kruif, A. and Maes, D. (2010a) Longitudinal field study to assess sow level risk factors associated with stillborn piglets. Anim. Reprod. Sci., 120(14): 78-83

4. Canario, L., Billon, Y., Caritez, J.C., Bidanel, J.P. and Laloë, D. (2009) Comparison of sow farrowing characteristics between a Chinese breed and three French breeds. Livest. Sci., 125(2-3): 132-140.

5. Borges, V.F., Bernardi, M.L., Bortolozzo, F.P. and Wentz, I. (2005) Risk factors for stillbirth and foetal mummification 
in four Brazilian swine herds. Prev. Vet. Med., 70(3-4): 165-176.

6. Le Cozler, Y., Guyomarc'h, C., Pichodo, X., Quinio, P.Y., and Pellois, H. (2002) Factors associated with stillborn and mummified piglets in high-prolific sows. Anim. Res., 51(3): 261-268

7. Mota-Rojas, D., Villanueva-Garcia, D., AlonsoSpilsbury, M., Becerril-Herrera, M., RamirezNecoechea, R., Gonzalez-Lozano, M. and TrujilloOrtega, M.E. (2007a) Effect of different doses of oxytocin at delivery on suffering and survival of newborn pigs. $J$. Med. Sci., 7(2): 170-178.

8. Mota-Rojas, D., Trujillo, M.E., Martinez, J., Rosales, A.M., Orozco, H., Ramirez, R., Sumano, H. and AlonsoSpilsbury, M. (2006) Comparative routes of oxytocin administration in crated farrowing sows and its effects on fetal and postnatal asphyxia. Anim. Reprod. Sci., 92(1-2): 123-143.

9. Mota-Rojas, D., Rosales, A.M., Trujillo, M.E., Orozco, H., Ramirez, R. and Alonso-Spilsbury, M. (2005c) The effects of vetrabutin chlorhydrate and oxytocin on stillbirth rate and asphyxia in swine. Theriogenology, 64(9): 1889-1897.

10. Mota-Rojas, D., Nava-Ocampo, A.A., Trujillo, M.E., Velazquez-Armenta, Y., Ramirez-Necoechea, R., MartinezBurnes, J. and Alonso-Spilsbury, Y.M. (2005b) Dose minimization study of oxytocin in early labor in sows: Uterine activity and fetal outcome. Reprod. Toxicol., 20(2): 255-259.

11. Mota-Rojas, D., Martinez-Burnes, J., Trujillo, M.E., Lopez, A., Rosales, A.M., Ramirez, R., Orozco, H., Merino, A. and Alonso-Spilsbury, M. (2005a) Uterine and fetal asphyxia monitoring in parturient sows treated with oxytocin. Anim. Reprod. Sci., 86(1-2): 131-141.

12. Canario, L., Cantoni, E., Le Bihan, E., Caritez, J.C., Billon, Y., Bidanel, J.P. and Foulley, J.L. (2006a) Betweenbreed variability of stillbirth and its relationship with sow and piglet characteristics. J. Anim. Sci., 84(12): 3185-3196.

13. Canario, L., Roy, N., Gruand, J. and Bidanel, J.P. (2006b) Genetic variation of farrowing kinetics traits and their relationships with litter size and perinatal mortality in French large white sows. J. Anim. Sci., 84(5): 1053-1058.

14. Jiarpinitnun, P., Loyawatananan, S., Sangratkanjanasin, P., Kompong, K., Nuntapaitoon, M., Muns, R., De Rensis, F. and Tummaruk, P. (2019) Administration of carbetocin after the first piglet was born reduced farrowing duration but compromised colostrum intake in newborn piglets. Theriogenology, 128(2019): 23-30.

15. Mota-Rojas, D., Villanueva-Garcia, D., VelazquezArmenta, E.Y., Nava-Ocampo, A.A., RamirezNecoechea, R., Alonso-Spilsbury, M. and Trujillo, M.E. (2007b) Influence of time at which oxytocin is administered during labor on uterine activity and perinatal death in pigs. Biol. Res., 40(1): 55-63.

16. Baxter, E.M., Jarvis, S., Sherwood, L., Robson, S.K., Ormandy, E., Farish, M., Smurthwaite, K.M., Roehe, R., Lawrence, A.B. and Edwards, S.A. (2009) Indicators of piglet survival in an outdoor farrowing system. Livest. Sci., 124(1-3): 266-276.

17. Hoai Nam, N. and Sukon, P. (2020) Risk factors associated with stillbirth in swine farms in Vietnam. World Vet. J., 10(1): 74-79.

18. Bhattarai, S., Framstad, T. and Nielsen, J.P. (2019) Association between sow and piglet blood hemoglobin concentrations and stillbirth risk. Acta. Vet. Scand., 61(1): 61.

19. Plush, K., Weaver, A. and Staveley, L. (2018) Maternal magnesium sulfate supplementation in a pre-farrow diet improves factors important for piglet viability. Animals
(Basel), 8(10): 185.

20. Bhattarai, S., Framstad, T. and Nielsen, J.P. (2018) Stillbirths in relation to sow hematological parameters at farrowing: A cohort study. J. Swine Health Prod., 26(4): 215-222.

21. Rangstrup-Christensen, L., Krogh, M.A., Pedersen, L.J. and Sorensen, J.T. (2017) Sow-level risk factors for stillbirth of piglets in organic sow herds. Animal, 11(6): 1078-1083.

22. Baxter, E.M., Jarvis, S., Palarea-Albaladejo, J. and Edwards, S.A. (2012) The weaker sex? The propensity for male-biased piglet mortality. PLoS One, 7(1): e30318.

23. Baxter, E.M., Jarvis, S., D'Eath, R.B., Ross, D.W., Robson, S.K., Farish, M., Nevison, I.M., Lawrence, A.B. and Edwards, S.A. (2008) Investigating the behavioural and physiological indicators of neonatal survival in pigs. Theriogenology, 69(6): 773-783.

24. Langendijk, P., Fleuren, M., van Hees, H. and van Kempen, T. (2018a) The course of parturition affects piglet condition at birth and survival and growth through the nursery phase. Animals (Basel), 8(5): 60.

25. Langendijk, P., Fleuren, M. and van Kempen, T.A. (2018b) Birth interval or duration of parturition: Which is relevant to risk of stillbirth and intervention? In: Proceedings of the Book of Abstracts of the $69^{\text {th }}$ Annual Meeting of the European Federation of Animal Science 2018. European Federation of Animal Science, Dubrovnik, Croatia. p27-31.

26. Alonso-Spilsbury, M., Mota-Rojas, D., Martínez-Burnes, J., Arch, E., López Mayagoitia, A., Ramírez-Necoechea, R., Olmos, A. and Trujillo, M.E. (2004) Use of oxytocin in penned sows and its effect on fetal intra-partum asphyxia. Anim. Reprod. Sci., 84(1-2): 157-167.

27. Paules, C., Dantas, A.P., Miranda, J., Crovetto, F., Eixarch, E., Rodriguez-Sureda, V., Dominguez, C., Casu, G., Rovira, C., Nadal, A., Crispi, F. and Gratacos, E. (2019) Premature placental aging in term small-for-gestational-age and growth-restricted fetuses. Ultrasound Obstet. Gynecol., 53(5): 615-622.

28. Zaleski, H.M. and Hacker, R.R. (1993) Variables related to the progress of parturition and probability of stillbirth in swine. Can. Vet. J., 34(2): 109-113.

29. Udomchanya, J., Suwannutsiri, A., Sripantabut, K., Pruchayakul, P., Juthamanee, P., Nuntapaitoon, M. and Tummaruk, P. (2019) Association between the incidence of stillbirths and expulsion interval, piglet birth weight, litter size and carbetocin administration in hyper-prolific sows. Livest. Sci., 227 (2019): 128-134.

30. Muglu, J., Rather, H., Arroyo-Manzano, D. and Bhattacharya, S. (2019) Risks of stillbirth and neonatal death with advancing gestation at term: A systematic review and meta-analysis of cohort studies of 15 million pregnancies. PLoS Med., 16(7): e1002838.

31. Deng, K., Huang, Y., Wang, Y., Zhu, J., Mu, Y., Li, X., Xing, A., Liu, Z., Li, M., Wang, X. and Liang, J. (2019) Prevalence of post-term births and associated maternal risk factors in China: Data from over 6 million births at health facilities between 2012 and 2016. Sci. Rep., 9(1): 273.

32. Middleton, P., Shepherd, E. and Crowther, C.A. (2018) Induction of labour for improving birth outcomes for women at or beyond term. Cochrane Database Syst. Rev., 5(5): Cd004945.

33. Maiti, K., Sultana, Z., Aitken, R.J., Morris, J., Park, F., Andrew, B., Riley, S.C. and Smith, R. (2017) Evidence that fetal death is associated with placental aging. Am. J. Obstet. Gynecol., 217(4): 441.e1-441.e14.

34. Vanderhaeghe, C., Dewulf, J., Jourquin, J., De Kruif, A. and Maes, D. (2011) Incidence and prevention of early parturition in sows. Reprod. Domest. Anim., 46(3): 428-433. 